\title{
An unusual presentation of ankylosing spondylitis
}

Singapore Med J 2020; 61(5): 283-284 https://doi.org/10.11622/smedj.2018080

Dear Sir,

Ankylosing spondylitis (AS), a relatively uncommon cause of low back pain, has significant implications for the individual.(1) AS has been profiled in the Singapore Chinese population, (2) and well-known radiological changes of the condition, particularly in the sacroiliac joints, have been documented. ${ }^{(3)}$ We herein present an unusual presentation of AS to the Sports Medicine Centre of Khoo Teck Puat Hospital, Singapore.

A 19-year-old national serviceman presented with bilateral hip pain that radiated to both knees. The hip pain had started two years earlier and gradually progressed to cause an antalgic gait with impaired mobility from associated muscle weakness. The pain was continuous and worsened with physical activity, and intermittent back discomfort was reported. He had no family history of inflammatory arthritis and had not undergone investigations or treatment even after multiple medical consultations with primary care and musculoskeletal physicians in the past.

Examination revealed weakness on squatting, pain on all range of movement of the hips and tenderness over both greater trochanters. There was normal range of movement of the back and no neurological deficit. An earlier ultrasonography examination had demonstrated gluteus medius tendinopathy, and rehabilitation was commenced for this problem. However, the patient's symptoms did not abate with rehabilitation, and pelvic magnetic resonance (MR) imaging was performed, which demonstrated florid enthesitis of the greater trochanter tendinous attachments bilaterally (worse on the right side), accompanied by mild left hip synovitis, joint effusion and acetabular subarticular oedema (Fig. 1a). Mild subarticular sacroiliac oedema was also demonstrated on MR imaging.

Following urgent communication of the findings, blood tests were requested, revealing elevated erythrocyte sedimentation rate $(65 \mathrm{~mm} / \mathrm{hr})$, low vitamin D level $(28.3 \mu \mathrm{g} / \mathrm{L})$ and a positive human leucocyte antigen B27 test. Subsequent whole-spine MR imaging identified early Romanus lesions of the lumbar spine, thus confirming the diagnosis of AS (Fig. 1b). A rheumatology referral was made, and a repeat physical examination with the modified Schober's test showed lumbar spine excursion of $3 \mathrm{~cm}$. As there was suboptimal improvement with nonsteroidal anti-inflammatory drugs alone, biologics treatment in the form of anti-tumour necrosis factor was commenced; significant improvements in pain, stiffness and mobility were seen within two months.

Hip involvement in AS has been more commonly documented in the juvenile form of AS (i.e. JAS). ${ }^{(4)}$ Indeed, it was estimated that $15 \%$ of patients had the classical axial skeletal presentation and $85 \%$ initially experienced oligoarthritis of the lower limbs. ${ }^{(5)}$ With its male preponderance (a ratio of 9:1) and peak incidence around 16 years of age, ${ }^{\left({ }^{6}\right)}$ our patient's presentation was in keeping with AS.

The present case highlights the importance of suspecting JAS, particularly when a patient presents with progressive lower limb pain and weakness around the pelvic area. Despite the multiple presentations to different practitioners over two years and the subsequent delay in arriving at a diagnosis, good communication between the multidisciplinary team expedited our patient's care. It has been suggested that the prevalence of hip involvement in AS has been underestimated. ${ }^{(7)}$ With the increased availability of MR imaging in medical care, we should perhaps consider this imaging modality as an earlier investigation when symptoms do not improve with conservative management, ${ }^{(8)}$ particularly when there is a risk of significant degenerative changes if interventions are not instigated early. ${ }^{(9)}$
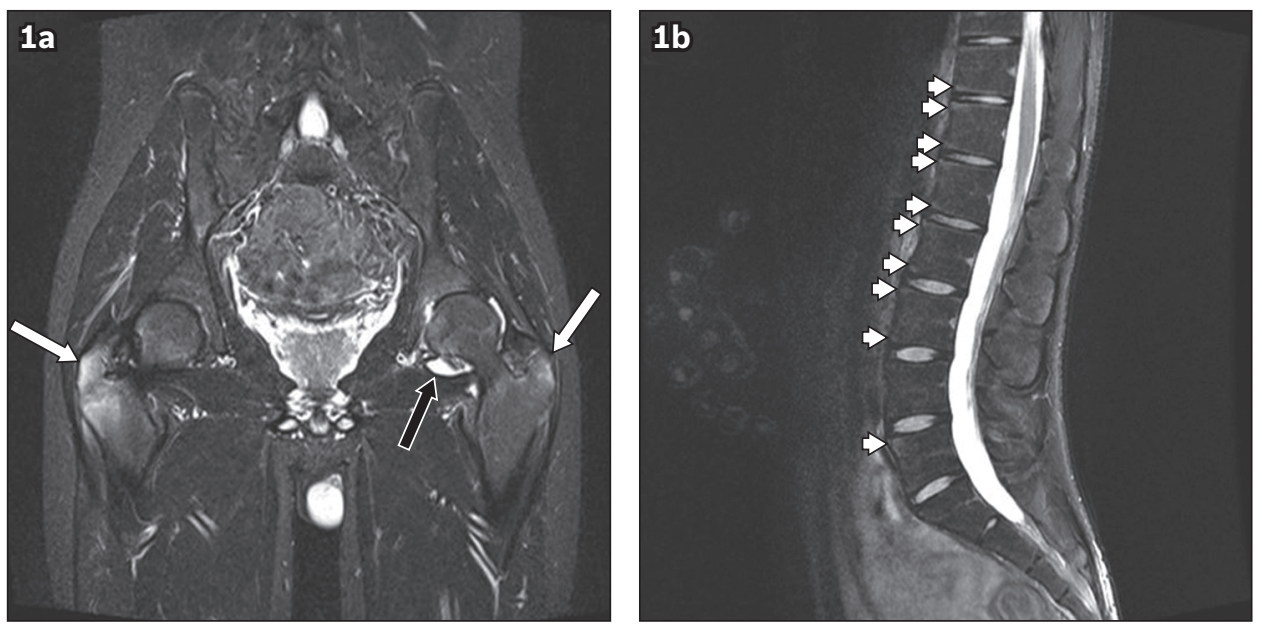

Fig. 1 (a) Coronal T2-W fat-suppressed MR image of the pelvis shows bilateral enthesitis with oedema in the greater trochanters, worse on the right (white arrows), with a small left hip joint effusion (black arrow). (b) Sagittal T2-W fat-suppressed MR image of the lumbar spine reveals early multilevel Romanus lesions (arrowheads). 


\section{Yours sincerely,}

Dinesh Sirisena' ${ }^{1}$, Yong-Yeow $\underline{\text { Chong }}^{2}$, Teck-Yew $\underline{\text { Chin }}^{3}$

${ }^{1}$ Sports Medicine Centre, Khoo Teck Puat Hospital, ${ }^{2}$ Raffles Internal Medicine Centre, Raffles Hospital, ${ }^{3}$ Department of Diagnostic Radiology, Khoo Teck Puat Hospital, Singapore.dinesh.sirisena@ktph.com.sg

\section{References}

1. Dean LE, Jones GT, MacDonald AG, et al. Global prevalence of ankylosing spondylitis. Rheumatology (Oxford) 2014; 53:650-7.

2. Koh WH, Howe HS, Boey ML. Ankylosing spondylitis in Singaporean Chinese - a clinical profile. Singapore Med J 1993; 34:518-20.

3. Peh WC. Clinics in diagnostic imaging (70). Bilateral sacroiliitis due to ankylosing spondylitis. Singapore Med J 2002; 43:107-11.

4. Mou Y, Zhang P, Li Q, et al. Clinical features in juvenile-onset ankylosing spondylitis patients carrying different B27 subtypes. BioMed Res Int 2015; 2015:594878.

5. Aggarwal A, Hissaria P, Misra R. Juvenile ankylosing spondylitis - is it the same disease as adult ankylosing spondylitis? Rheumatol Int 2005; 25:94-6.

6. Singh G, Lawrence A, Agarwal V, Misra R, Aggarwal A. Higher prevalence of extra-articular manifestations in ankylosing spondylitis with peripheral arthritis. J Clin Rheumatol 2008;14:264-6.

7. Huang ZG, Zhang XZ, Hong W, et al. The application of MR imaging in the detection of hip involvement in patients with ankylosing spondylitis. Eur J Radiol 2013; 82:1487-93.

8. Chen D, Yuan S, Zhan Z, et al. Early-stage hip involvement in patients with ankylosing spondylitis: a Chinese study based on magnetic resonance imaging. Mod Rheumatol 2016; 26:933-9.

9. Chen HA, Chen $\mathrm{CH}$, Liao HT, et al. Factors associated with radiographic spinal involvement and hip involvement in ankylosing spondylitis. Semin Arthritis Rheum $2011 ; 40: 552-8$ 